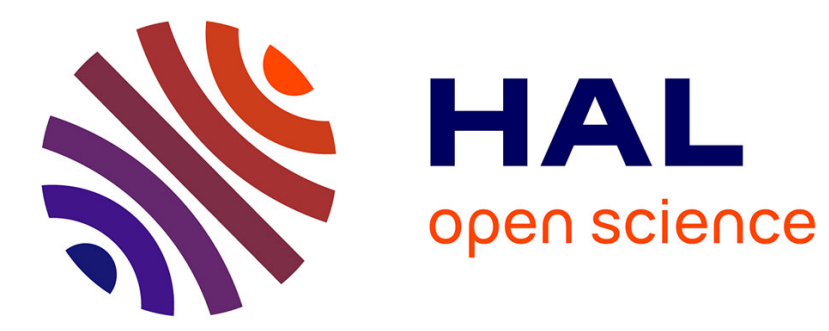

\title{
Spin-transfer-torque reversal in perpendicular anisotropy spin valves with composite free layers
}

I Yulaev, M V Lubarda, S. Mangin, V. Lomakin, Eric E. Fullerton

\section{To cite this version:}

I Yulaev, M V Lubarda, S. Mangin, V. Lomakin, Eric E. Fullerton. Spin-transfer-torque reversal in perpendicular anisotropy spin valves with composite free layers. Applied Physics Letters, 2011, 99 (13), pp.132502. 10.1063/1.3643046 . hal-02085966

\section{HAL Id: hal-02085966 https://hal.univ-lorraine.fr/hal-02085966}

Submitted on 1 Apr 2019

HAL is a multi-disciplinary open access archive for the deposit and dissemination of scientific research documents, whether they are published or not. The documents may come from teaching and research institutions in France or abroad, or from public or private research centers.
L'archive ouverte pluridisciplinaire HAL, est destinée au dépôt et à la diffusion de documents scientifiques de niveau recherche, publiés ou non, émanant des établissements d'enseignement et de recherche français ou étrangers, des laboratoires publics ou privés. 


\title{
Spin-transfer-torque reversal in perpendicular anisotropy spin valves with composite free layers
}

\author{
I. Yulaev, ${ }^{1,2}$ M. V. Lubarda, ${ }^{1,2}$ S. Mangin, ${ }^{3}$ V. Lomakin, ${ }^{1,2}$ and Eric E. Fullerton ${ }^{1,2, a)}$ \\ ${ }^{1}$ Center for Magnetic Recording Research, University of California, San Diego, La Jolla, \\ California 92093, USA \\ ${ }^{2}$ Department of Electrical and Computer Engineering, University of California, San Diego, \\ La Jolla, California 92093, USA \\ ${ }^{3}$ Institut Jean Lamour, CNRS, Nancy Université, UPV Metz, Vandoeuvre les Nancy, France
}

(Received 6 June 2011; accepted 25 August 2011; published online 26 September 2011)

We describe modeling of spin-transfer-torque (STT) driven reversal in nanopillars with strong out-of-plane magnetic anisotropy where the free layer is a magnetically hard-soft composite structure. By adjusting the exchange coupling between the hard and soft layers, we observed reduced current amplitude and pulse durations required to reverse the magnetization compared to a homogeneous free layer of comparable thermal stability. The reduction in critical current comes from the increased STT efficiency acting on the soft layer. As such, the switching current is relatively insensitive to the damping parameter of the magnetic hard layer. These properties make composite free layers promising candidates for STT-based magnetic memories. (C) 2011 American Institute of Physics. [doi:10.1063/1.3643046]

Spin-transfer-torque (STT) based magnetic random access memories (MRAM) are a promising technologies for implementing non-volatile storage in commercial integrated circuits. ${ }^{1}$ One of the present challenges in implementing STT-MRAM is the reduction in critical current $\left(I_{C}\right)$, the current required to change the magnetization of the free memory element while maintaining a sufficient thermal stability for non-volatile applications. A reduction in $\mathrm{I}_{\mathrm{C}}$ is essential for reducing power dissipation and current.

STT devices exhibiting perpendicular anisotropy provide a pathway to low critical current and high thermal stability. $^{2}$ In the perpendicular geometry, the critical current for the onset of spin-torque reversal for a macrospin is given by

$$
I_{C 0}=\left(\frac{2 e}{\hbar}\right) \frac{2 \alpha}{\eta(\theta) p} E_{B}
$$

assuming zero temperature and no applied fields. Here, $E_{B}$ is the energy barrier for reversal, $\alpha$ is the Gilbert damping constant of the free layer, $\eta(\theta)$ is the angular dependence of spin torque transfer efficiency, and $p$ is the spin polarization of the current. As $E_{B}$ is set by the thermal stability requirements of the device (typically $E_{B}>50 \mathrm{k}_{\mathrm{B}} \mathrm{T}$ ), further reductions require decreasing $\alpha$ and increasing $\eta(\theta) p$ as in recent demonstrations of STT switching of perpendicular anisotropy $\mathrm{CoFeB} / \mathrm{MgO} / \mathrm{CoFeB}$ tunnel junctions. ${ }^{3}$ For current pulses of finite time $(\tau)$, modeling and experiments of fast time switching of perpendicular anisotropy nanopillars show that

$$
\tau^{-1}=A\left(I_{C}-I_{C 0}\right),
$$

where $I_{C}$ is the current required to reverse the magnetization and the parameter $A$ governs the switching rate. ${ }^{4,5}$

In this letter, we describe STT-driven switching of exchange coupled magnetically hard/soft bi-layer as the free layer. Such

\footnotetext{
${ }^{\text {a) }}$ Author to whom correspondence should be addressed: Electronic mail: efullerton@ucsd.edu.
}

composite structures have been extensively studied for their efficiency for magnetic field switching, particularly for magnetic recording $^{6-9}$ and for microwave assisted magnetic recording. ${ }^{10}$ The model used in the present calculation is schematically depicted in Fig. 1. The reference layer is fixed in the calculations. The free layer has a relatively soft layer \#1 that interacts with the reference layer via the STT interactions. The soft layer is ferromagnetically exchange coupled $\left(J_{e x}=0.2-5 \mathrm{ergs} / \mathrm{cm}^{2}\right)$ to the magnetically harder layer \#2 having relatively higher magneto-crystalline anisotropy. ${ }^{11,12}$ For the examples discussed in this paper, the soft layer has identical parameters as the hard layer, except with magneto-crystalline anisotropy $K_{U}$ reduced by a factor of $\sim 7$ relative to the hard layer. We further alter the damping parameters of hard and soft layers for selected calculations.

We run time-domain simulations of both current and field induced switching dynamics for the composite free layer by solving the Landau-Lifshitz-Gilbert (LLG) equation modified to include spin-torque term. We assumed that there is no spin torque interaction between layers \#1 and \#2 such that the spin-torque interaction from the reference layer only affects layer \#1 of the composite layer. We have performed both macrospin (where each layer of the composite is treated as a macrospin) and micromagnetic simulations and have verified that the results are self-consistent. The micromagnetic simulations used the LLG Micromagnetics Simulator where the magnetic layers were discretized into $4 \times 4 \mathrm{~nm}^{2}$ squares. For both simulations, we assume the symmetric Slonczewski approximation for $\eta(\theta)=\frac{q}{A_{S S}+B_{S S} \cos (\theta)}$, where $A_{S S}$ and $B_{S S}$ are functions of polarization $P$ that approximate a metallic spacer. ${ }^{13,14}$ We also performed macrospin calculations using $\eta(\theta)=\eta_{0}$ which more closely represents a tunnel junction.

An example time trace showing reversal of the composite free layer is shown in Fig. 1 for $J_{e x}=1 \mathrm{erg} / \mathrm{cm}^{2}$ and $I=433 \mu \mathrm{A}$. As a result of the finite exchange between the hard and soft layers, the softer layer (dashed line) responds more strongly to the STT interaction as compared to the hard 


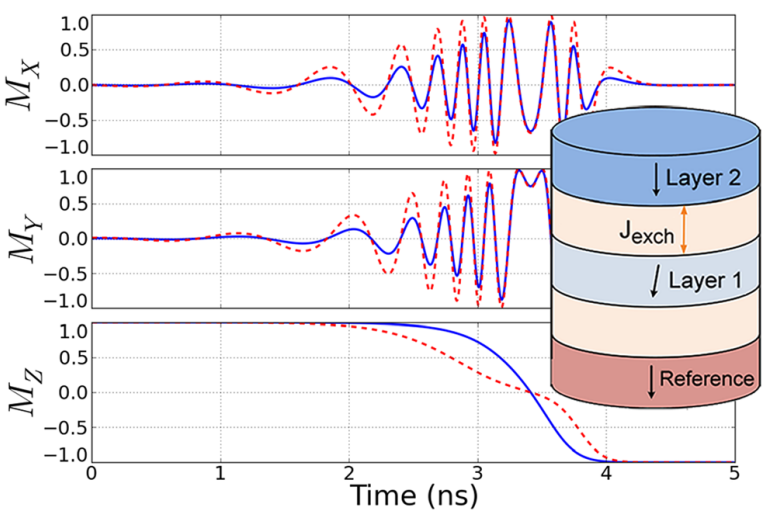

FIG. 1. (Color online) STT switching results of a composite free layer structures (inset) that is made up of two ferromagnetic layers that are ferromagnetically exchange coupled. The lower anisotropy layer 1 interacts with the reference layer via the STT interactions. The time traces show the three components of magnetization for layers 1 (dashed) and 2 (solid) during reversal where $\mathrm{M}_{\mathrm{z}}$ is normal to the layers.

layer (solid line) leading to larger precession angles prior to reversal. The soft layer initializes reversal as seen most clearly in the $\mathrm{M}_{\mathrm{z}}$ scan. This behavior is similar to that observed in microwave assisted magnetic recording simulations for composite structures. ${ }^{10}$ To quantify the efficiency of this approach for spin-torque reversal, we compare the dependence of $E_{B}$, the coercive field $H_{C}$, and the critical current for reversal $\left(I_{C}\right)$ on the interlayer exchange coupling (Fig. 2). Each quantity is normalized to the values for large $J_{e x}$ where the free layer can be considered a single macrospin.

We calculate $E_{B}$ for the composite system using an approach described in Ref. 15. $E_{B}$ is only weakly dependent on the exchange coupling (Fig. 2(a)) decreasing less than $10 \%$ down to $J_{e x}=0.4 \mathrm{ergs} / \mathrm{cm}^{2}$. We also see a decrease in $H_{C}$ of the free layer (Fig. 2(a)) with decreasing exchange coupling from the strong-coupling limit until a minimum is reached at approximately $2.5 \mathrm{ergs} / \mathrm{cm}^{2}$ and then increases quickly with further decrease of the coupling. This behavior, in agreement with previous experimental data ${ }^{16}$ and theoretical modeling, ${ }^{17,18}$ results from incoherent reversal of the composite structure.

A similar but enhanced behavior is obtained for STT switching. We assume square write pulses of duration $(\tau)$ and determine the minimum current for reversal. Results for $\tau=20 \mathrm{~ns}$ and $\tau=2$ ns are shown in Figs. 2(a) and 2(b), respectively. The soft layer is initiated with a $\sim 0.6^{\circ}$ tilt in magnetization away from the easy axis. There are strong reductions in $I_{C}$ with reduced coupling strength that depend on both the functional form of $\eta(\theta)$ and $\tau$. For the constant $\eta(\theta)$, the parallel-to-antiparallel (P-to-AP) and AP-to-P switching are equivalent, and we see a $50 \%$ and $40 \%$ reduction of the critical current relative the strong coupling limit for 20 and 2 ns, respectively. For the Slonczewski form of $\eta(\theta)$, there is a strong difference between P-to$\mathrm{AP}$ and AP-to-P switching, particularly at longer pulse times where the composite structure is more effective in reducing the P-to-AP current for low coupling strengths. As the P-to-AP switching is the least efficient, i.e., requiring greater current to induce switching of the magnetization, this reduces the asymmetry when comparing $\mathrm{I}_{\mathrm{C}}$ for P-to-AP versus AP-to-P switching.

This difference in AP-to-P and P-to-AP switching is also seen in micromagnetic calculations (Fig. 3) where we

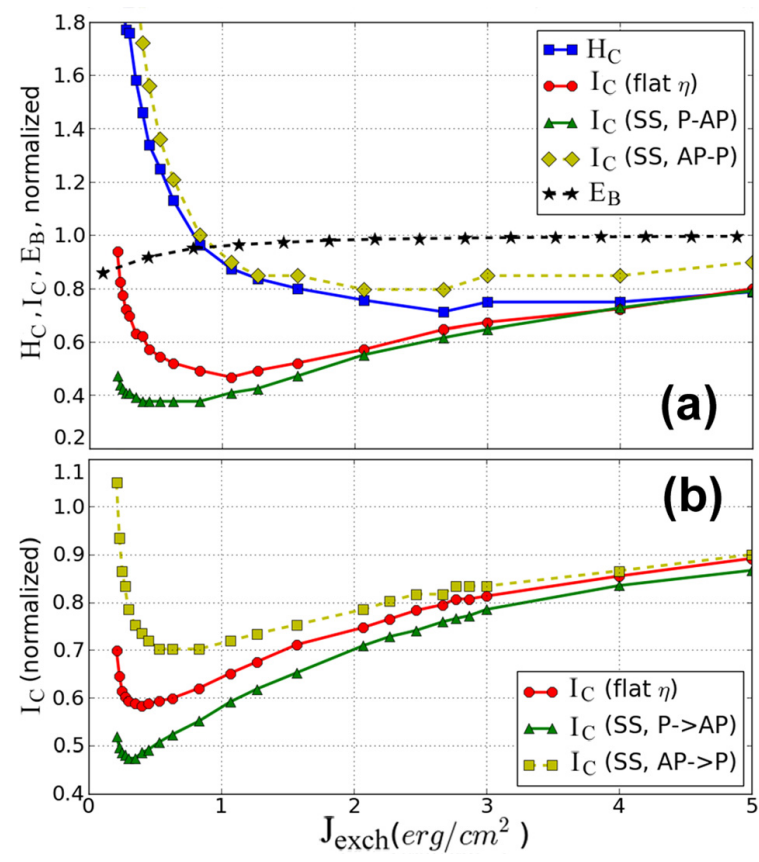

FIG. 2. (Color online) Switching field $\left(H_{C}\right)$, switching current $\left(I_{C}\right)$, and energy barrier $\left(E_{B}\right)$ results for a composite free layer as a function of ferromagnetic exchange coupling $\left(J_{e x}\right)$ in the composite free layer. All quantities are normalized to the values for large $J_{e x}$. The values for $H_{C}$ and $E_{B}$ are given in (a). $I_{C}$ values are given both AP-to-P and P-to-AP switching for the symmetric Slonczewski (SS) approximation for $\eta(\theta)$ and assuming a flat $\eta(\theta)=\eta_{0}$. The values for a 20-ns current pulses are given in (a) and 2-ns pulses in (b).

calculate $I_{C}$ vs. $\tau$ for $J_{e x}=1 \mathrm{ergs} / \mathrm{cm}^{2}$ compared to the reference model where $J_{e x}=10 \mathrm{ergs} / \mathrm{cm}^{2}$. In the simulation, we use an AP reference layer to minimize the dipolar interaction of the reference and free layers. ${ }^{12}$ The results in Fig. 3 are plotted as $1 / \tau$ vs. $I_{C}$. The simulated results generally follow the expectations of Eq. (2) and only deviate somewhat for the shortest pulse durations. For switching from AP to P, we observe only a small change of $I_{C 0}$ (the X-axis intercept) but an increase of a factor 2 in the $A$ parameter for the composite structure. This results in significantly reduced current at finite pulse widths reaching a 50\% reduction in the critical current at $1 \mathrm{~ns}$. For P-to-AP switching, we see a roughly two-fold decrease in $I_{C O}$ for the coupled bi-layer in addition to modest increase in A. Again, we see a roughly $50 \%$ decrease in the switching current at $1 \mathrm{~ns}$. For both orientations, we observe that the critical current is more strongly reduced compared to the reduction in $E_{B}$. While the general trends observed in Fig. 3 are maintained for different $J_{e x}$ (and also observed in macrospin calculations), the detailed behavior of $I_{C 0}$ and $A$ depend on the interlayer coupling.

The general trends shown in Figs. 1-3 can be understood from the angular dependence of the spin-torque interaction. The soft layer moves out of the P or AP orientation relative to the reference layer much faster than the hard layer (Fig. $1)$. As the soft free layer moves, there is an increase in the spin-torque $\mathbf{N}_{\text {st }}$ which depends on $\eta(\theta) \sin (\theta) .{ }^{14}$ For the symmetric Sloncewski form of $\eta(\theta)$, there is a stronger increase in $\mathbf{N}_{\mathrm{st}}$ with angle for AP-to-P switching. Only small angles are needed for the soft layer to effectively pull the hard layer and the optimum coupling is relatively strong (Fig. 1(a)). 


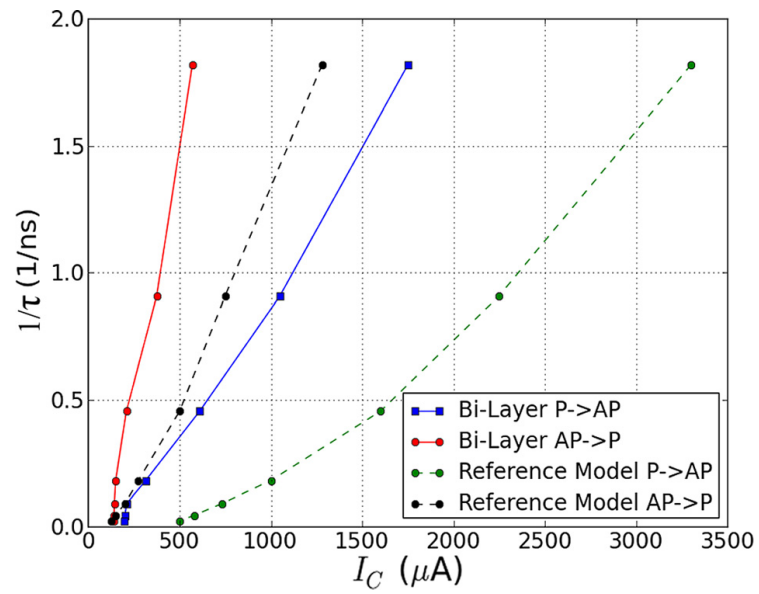

FIG. 3. (Color online) Micromagnetic results for switching critical currents of a composite free layer for varying current pulse durations $(\tau)$ assuming a symmetric Slonczewski approximation for $\eta(\theta)$. Results are shown both for a $J_{e x}=1 \mathrm{ergs} / \mathrm{cm}^{2}$ and a reference calculations where $J_{e x}=10 \mathrm{ergs} / \mathrm{cm}^{2}$.

Conversely, for P-to-AP switching, the increase in $\mathbf{N}_{\mathrm{st}}$ is weaker such that larger deviations of the soft layer are needed and the optimum coupling is weaker so that the composite layer is more effective. The case for a constant $\eta(\theta)$ is in between these limits.

An additional benefit may arise using a composite bilayer when $\alpha$ is unequal in the soft and hard layers. The damping of the soft layer plays a greater role in determining the effective damping of the structure and hence the switching current. Using the macrospin model, we simulate the switching behavior of the composite bilayer for different combinations of damping parameter values (Fig. 4). For these calculations, we use a relatively large $\tau=100 \mathrm{~ns}$ such that $I_{C}$ is dominated by $I_{C 0}$. If we increase the damping in both layers, $I_{C}$ is proportional to the damping as expected from Eq. (1). Increasing either the damping parameter of the soft layer $\left(\alpha_{\text {soft }}\right)$ or the hard layer $\left(\alpha_{\text {hard }}\right)$ while holding the other layer fixed at 0.01 also yields a linear increase in $I_{C}$. However, the slope is shallower when increasing the damping in the harder layer. For example shown in Fig. 4, a 10-fold increase in $\alpha_{\text {soft }}$ yields a 7 -fold increase in $I_{C}$ where a 10 -fold increase in $\alpha_{\text {hard }}$ yields only a

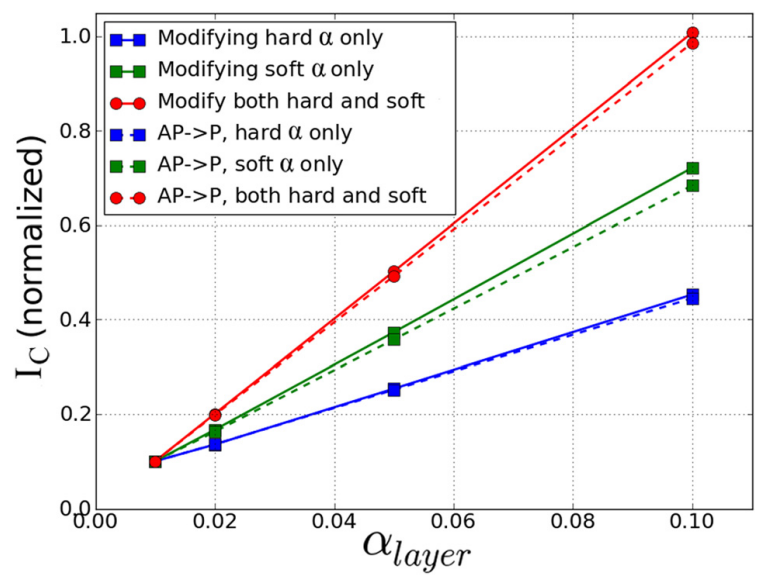

FIG. 4. (Color online) Changes in the critical current as a function of damping parameter in the hard, soft, or both layers. The critical current is normalized to value for $\alpha_{\text {soft }}=\alpha_{\text {hard }}=0.1$. 4.50-fold increase in $I_{C}$. This property may have important practical applications as many recent experiments have shown $\alpha$ and $K_{U}$ to be positively correlated. . $^{3,19-24}$

In conclusion, we described modeling of spin-transfer-torque reversal in nanopillars with strong out-of-plane magnetic anisotropy where the free layer is a magnetically hard-soft composite structure. We find for modest coupling between the hard and soft layers that there is a reduction in $I_{C 0}$ and an increase in $A$ which results in improved switching efficiency without a corresponding reduction in $\mathrm{E}_{\mathrm{B}}$. The reduction in critical current comes from the increased STT efficiency acting on the soft layer combined with incoherent reversal of the composite structure. As such, the switching current is relatively insensitive to the damping parameter of the magnetic hard layer. These properties make these structures promising candidates for spintransfer-torque based magnetic memories.

We would like to thank A. Kent for helpful discussions. This work is supported by NSF Award No. DMR-1008654 and by the Friends contract of the French National Research Agency (ANR).

${ }^{1}$ J. A. Katine and E. E. Fullerton, J. Magn. Magn. Mater. 320, 1217 (2008). ${ }^{2}$ S. Mangin, Y. Henry, D. Ravelosona, J. A. Katine, and E. E. Fullerton, Appl. Phys. Lett. 94, 012502 (2009).

${ }^{3}$ S. Ikeda, K. Miura, H. Yamamoto, K. Mizunuma, H. D. Gan, M. Endo, S. Kanai, J. Hayakawa, F. Matsukura, and H. Ohno, Nature Mater. 9, 721 (2010).

${ }^{4}$ D. Bedau, H. Liu, J. J. Bouzaglou, A. D. Kent, J. Z. Sun, J. A. Katine, E. E. Fullerton, and S. Mangin, Appl. Phys. Lett. 96, 022514 (2010).

${ }^{5}$ D. Bedau, H. Liu, J. Z. Sun, J. A. Katine, E. E. Fullerton, S. Mangin, and A. D. Kent, Appl. Phys. Lett. 97, 262502 (2010).

${ }^{6}$ N. F. Supper, D. T. Margulies, A. Moser, A. Berger, H. Do, and E. E. Fullerton, IEEE Trans. Magn. 41, 3238 (2005).

${ }^{7}$ E. E. Fullerton, H. V. Do, D. T. Margulies, and N. Supper, U.S. patent 7,425,377 (Sept. 16, 2008).

${ }^{8}$ R. H. Victora and X. Shen, IEEE Trans. Magn. 41, 2828 (2005).

${ }^{9}$ D. Suess, T. Schrefl, S. Fahler, M. Kirschner, G. Hrkac, F. Dorfbauer, and J. Fidler, Appl. Phys. Lett. 87, 12504 (2005).

${ }^{10} \mathrm{~S}$. Li, B. Livshitz, H. N. Bertram, M. Schabes, T. Schrefl, E. E. Fullerton, and V. Lomakin, Appl. Phys. Lett. 94, 202509 (2009).

${ }^{11}$ Antiferromagnetically (AF)-coupled free layers are less efficient that ferromagntically coupled free layers. In the AF-coupled case, the preferred precession direction for the harder layer (\#2) is counterclockwise to the direction of the anisotropy field, whereas the softer layer (\#1) wishes to precess counterclockwise to exchange field coming from layer \#2 (which is opposite to the direction of the hard layer anisotropy field for AF coupling). Thus, the two layers wish to precess with opposite cyclicity, but the exchange coupling opposes this dynamic suppressing reversal consistent with experimental observations (Ref. 12)

${ }^{12}$ I. Tudosa, J. A. Katine, S. Mangin, and E. E. Fullerton, Appl. Phys. Lett. 96, 212504 (2010)

${ }^{13}$ J. C. Slonczewski, J. Magn. Magn. Mater. 159, L1 (1996).

${ }^{14}$ J. Xiao, A. Zangwill, and M. D. Stiles, Phys. Rev. B 72, 014446 (2005).

${ }^{15}$ W. J. Chen, S. F. Zhang, and H. N. Bertram, J. Appl. Phys. 71, 5579 (1992).

${ }^{16}$ T. Hauet, E. Dobisz, S. Florez, J. Park, B. Lengsfield, B. D. Terris, and O. Hellwig, Appl. Phys. Lett. 95, 262504 (2009).

${ }^{17}$ H. N. Bertram and B. Lengsfield, IEEE Trans. Magn. 43, 2145 (2007).

${ }^{18}$ T. P. Nolan, B. F. Valcu, and H. J. Richter, IEEE Trans. Magn. 47, 63 (2011).

${ }^{19}$ A. Barman, S. Wang, O. Hellwig, A. Berger, E. E. Fullerton, and H. Schmidt, J. Appl. Phys. 101, 09D102 (2007).

${ }^{20}$ J. M. Beaujour, D. Ravelosona, I. Tudosa, E. E. Fullerton, and A. D. Kent, Phys. Rev. B 80, 180415 (2009).

${ }^{21}$ E. P. Sajitha, J. Walowski, D. Watanabe, S. Mizukami, F. Wu, H. Naganuma, M. Oogane, Y. Ando, and T. Miyazaki, IEEE Trans. Magn. 46, 2056 (2010).

${ }^{22}$ S. Mizukami, E. P. Sajitha, D. Watanabe, F. Wu, T. Miyazaki, H. Naganuma, M. Oogane, and Y. Ando, Appl. Phys. Lett. 96, 152502 (2010).

${ }^{23}$ N. Fujita, N. Inaba, F. Kirino, S. Igarashi, K. Koike, and H. Kato, J. Magn. Magn. Mater. 320, 3019 (2008).

${ }^{24}$ S. Pal, B. Rana, O. Hellwig, T. Thomson, and A. Barman, Appl. Phys. Lett. 98, 082501 (2011). 